\title{
Treatment of Early Breast Cancer Patients: Evidence, Controversies, Consensus: Focusing on Systemic Therapy - German Experts' Opinions for the 16th International St. Gallen Consensus Conference (Vienna 2019)
}

\author{
Diana Lüftner $^{\mathrm{a}}$ Ingo Bauerfeind ${ }^{\mathrm{b}}$ Michael Braun ${ }^{\mathrm{c}}$ Sara Y. Brucker ${ }^{\mathrm{d}}$ Peter A. Fasching ${ }^{\mathrm{e}}$ \\ Ricardo Felberbaum $^{f}$ Friederike Hagemann ${ }^{g}$ Renate Haidinger ${ }^{\text {h }}$ Nadia Harbeck ${ }^{g}$

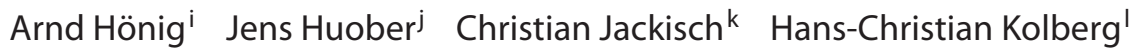 \\ Cornelia Kolberg-Liedtke $^{m}$ Thorsten Kühn ${ }^{n} \quad$ Nicolai Maass $^{\circ} \quad$ Toralf Reimer $^{p}$ \\ Andreas Schneeweiss $^{q} \quad$ Eva Schumacher-Wulf $^{r}$ Florian Schütz $^{\mathrm{s}} \quad$ Christoph Thomssen $^{\mathrm{t}}$ \\ Michael Untch ${ }^{\mathrm{u}}$ Rachel Wuerstlein ${ }^{\mathrm{g}}$ Marc Thill $^{v}$ \\ aDepartment of Hematology, Oncology and Tumour Immunology, Charité - Campus Benjamin Franklin, Berlin,

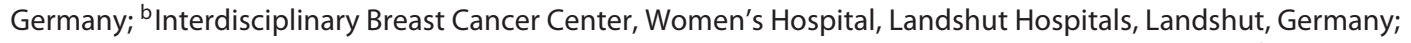 \\ 'Interdisciplinary Breast Center, Women's Hospital, Red Cross Hospital Munich, Munich, Germany; ${ }^{\text {dUniversity }}$ \\ Women's Hospital Tübingen, Tübingen, Germany; ${ }^{~}$ Women's Hospital at the University Hospital Erlangen, \\ Comprehensive Cancer Center Erlangen-EMN, Friedrich-Alexander University Erlangen-Nuremberg, Erlangen, \\ Germany; ${ }^{\mathrm{f}}$ Hospital Association Kempten-Oberallgäu, Kempten Hospitals, Kempten, Germany; ${ }^{\mathrm{g} B r e a s t}$ Center, \\ Women's Hospital at the Ludwig Maximillian University Munich (LMU), Munich, Germany; ${ }^{\mathrm{h}}$ Brustkrebs Deutschland \\ e.V., Hohenbrunn, Germany; ${ }^{~}$ Breast Center, Women's Hospital, Catholic Hospital Mainz, Mainz, Germany; ${ }^{j}$ Breast

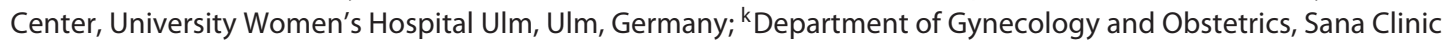 \\ Offenbach, Offenbach, Germany; 'Breast Center, Department of Gynecology and Obstetrics, Marienhospital Bottrop \\ gGmbH, Bottrop, Germany; ${ }^{\mathrm{m}}$ Charité - Campus Mitte, University Hospital Berlin, Berlin, Germany; ${ }^{\mathrm{n}}$ Department of \\ Gynecology and Obstetrics, Esslingen Hospitals, Esslingen, Germany; ${ }^{\circ}$ Breast Center, Department of Gynecology

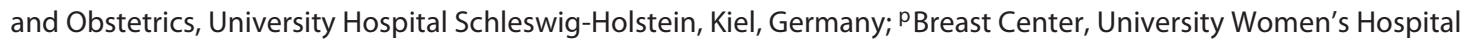 \\ and Outpatient Clinic, Clinics Südstadt Rostock, Rostock, Germany; ${ }^{9}$ National Centrum for Tumor Disease (NCT), \\ University Hospital (UKHD), and German Cancer Research Site (DKFZ), Heidelberg, Germany; ${ }^{r}$ Mamma Mia! Das \\ Brustkrebsmagazin, Kronberg, Germany; ${ }^{5}$ Senology Department, Breast Center, University Hospital in Heidelberg,

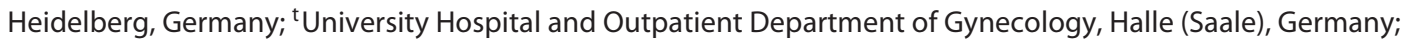 \\ uDepartment of Gynecology and Obstetrics, Interdisciplinary Breast Center, HELIOS Hospitals Berlin Buch, Berlin, \\ Germany; ${ }^{\vee}$ Department of Gynecology and Obstetrics, Interdisciplinary Breast Center, Agaplesion Markus Hospital, \\ Frankfurt/M., Germany
}

\section{Keywords}

St. Gallen Consensus Conference 2019 • Early breast cancer . Locoregional therapy · Axilla · Neoadjuvant treatment · Systemic therapy

D. Lüftner, S.Y. Brucker, P.A. Fasching, N. Harbeck, J. Huober, C. Jackisch, C. Kolberg-Liedtke, T. Kühn, A. Schneeweiss, C. Thomssen, and M. Untch are part of the Writing Committee. S.Y. Brucker, N. Harbeck, and J. Huober are St. Gallen Panel Experts.

\section{KARGER}

() 2019 S. Karger AG, Basel

\begin{abstract}
A German working group of leading breast cancer experts have discussed the votes at the International St. Gallen Consensus Conference in Vienna for the treatment of primary breast cancer with regard to the German AGO (Arbeitsgemeinschaft Gynäkologische Onkologie) recommendations for clinical practice in Germany. Three of the German breast cancer experts were also members of this year's St. Gallen panel. Comparing the St. Gallen recom-
\end{abstract}


mendations with the annually updated treatment recommendations of the Gynecological Oncology Working Group (AGO Mamma 2019) and the German S3 Guideline is useful, because the recommendations of the St. Gallen panel are based on expert opinions of different countries and disciplines. The focus of this article is on systemic therapy. The motto of this year's 16th St. Gallen Consensus Conference was "Estimating the magnitude of clinical benefit." The rationale behind this motto is that, for every treatment decision, a benefit-risk assessment must be taken into consideration for each patient.

() 2019 S. Karger AG, Basel

\section{Introduction}

The objective of the St. Gallen Consensus Conference on "Primary Treatment of Early Breast Cancer" in Vienna was to establish an international consensus on the treatment of patients with early - nonmetastatic - breast cancer in daily clinical practice. The consensus is based on the voting outcomes of the St. Gallen panel on current clinical questions. This year's panel consisted of more than 50 breast cancer experts from 23 countries, including 5 German experts (Table 1). The panelists not only represent different countries with different healthcare systems and resources, but as a result of the interdisciplinary nature of treatment, they also represent different areas of expertise.

Against this background, for several years, a German working group has been reviewing the voting results of the St. Gallen panelists on the basis of the German guidelines $[1,2]$ and, specifically, the annually updated treatment recommendations of the Gynecological Oncology Working Group, section "Mamma" (AGO [Arbeitsgemeinschaft Gynäkologische Onkologie] Mamma) [1].

\section{Thematic Focus of the St. Gallen Consensus}

This year's 16th St. Gallen Consensus Conference had the motto "Estimating the magnitude of clinical benefit of local and systemic therapies in patients with early breast cancer." The aim was to match the treatment decision to the individual situation of patients with early breast cancer. Against the background of evidencebased data, a benefit-risk assessment is necessary to decide under which conditions treatment is appropriate and when not (or no longer) in every individual patient.

The voting questions included local and locoregional as well as the systemic treatment of early breast cancer. This article focuses on systemic therapy. Relating to questions concerning surgery and radiotherapy, we would like to refer to a different publication [3]. We also refer to the initial report in Breast Care focusing on this year's St. Gallen Consensus Conference in Vienna [4].

\section{Significance of Tumor-Infiltrating Lymphocytes and Programmed Cell Death Ligand 1 Status}

In the context of systemic treatment, pathology is becoming increasingly important for treatment decision. Further predictive factors are discussed in addition to hormone receptor (HR) and HER2 status, such as the role of tumor-infiltrating lymphocytes (TILs) or the programmed cell death ligand 1 (PD-L1) status.

For early triple-negative breast cancer (TNBC), the majority of the St. Gallen panelists voted in favor of routinely assessing TILs. German experts do not agree with this, because there are currently no therapeutic consequences from measuring TILs. In the opinion of the German experts, a high TIL percentage does not imply that chemotherapy can be omitted.

The first PD-L1 inhibitor (atezolizumab) has already been approved in the US and in Europe in combination with nab-paclitaxel for the treatment of patients with metastatic PD-L1-positive TNBC. The panelists and German experts stated that this has no impact on early TNBC. Currently, there are no mature study data available on a $\mathrm{PD} /$ PD-L1 inhibitor in early TNBC. Neither the St. Gallen panelists (79.2\%) nor the German expert group, therefore, consider that routine PD-L1 determination in early TNBC is indicated. This also applies to PD-L1 determination on the immune cells (majority vote: $91.5 \%$ ), which supports the treatment indication in the metastatic setting $[5,6]$.

\section{Impact of Multigene Signatures}

Multigene signatures may support the decision to treat with or without chemotherapy in patients with $\mathrm{ER}+$ / HER2 early breast cancer. A majority of more than $90 \%$ of the St. Gallen panelists recommend the use of multigene expression analyses for patients with $\mathrm{T} 1 / 2$ breast cancer without lymph node involvement (93.6\%), and a majority also recommend this for patients with T3N0 cancer $(74.5 \%)$ and patients with $1-3$ positive lymph nodes regardless of the $\mathrm{T}$ stage $(78.7 \%)$.

From the German point of view, multigene expression analyses should only be used when the classic clinical parameters leave doubts about whether a chemotherapy is indicated or not (Fig. 1) [1]. In patients with lymph node involvement, the German experts consider that multigene expression analyses play no role in routine, because their clinical value has not been validated in prospective studies in these patients thus far. The German expert group also refers to the recommendations of the AGO Mamma, ac- 
Table 1. International St. Gallen Panel 2019 - International St. Gallen Consensus Panel Participants

Chair: Giuseppe Curigliano (Italy), Eric P. Winer (USA)

Consensus Writing Committee: Harold J. Burstein (USA), Marco Colleoni (Italy), Giuseppe Curigliano (Italy), Carsten Denkert (Germany), Peter Dubsky (Austria/Switzerland), Michael Gnant (Austria),

Sibylle Loibl (Germany), Martine Piccart-Gebhart (Belgium), Philip Poortmans (Netherlands/France), Meredith Regan (USA), Hans-Jörg Senn (Switzerland), Beat Thürlimann (Switzerland), Eric P. Winer (USA)

\begin{tabular}{|c|c|c|}
\hline Harold J. Burstein (USA) & Viviana Galimberti (Italy) & Philip Poortmans \\
\hline Fabrice André (France) & Heba Gamal (Egypt) & (Netherlands/France) \\
\hline Jonas Bergh (Sweden) & Judy Garber (USA) & Kathleen Pritchard (Canada) \\
\hline Hervé Bonnefoi (France) & William J. Gradishar (USA) & Meredith Regan (USA) \\
\hline Sara Y. Brucker (Germany) & Karen Gelmon (Canada) & Emiel J.T. Rutgers (Netherlands) \\
\hline Fatima Cardoso (Portugal) & Bahadir Gulluoglu (Turkey) & Felix Sedlmayer (Austria) \\
\hline Lisa Carey (USA) & Nadia Harbeck (Germany) & Vladimir Semiglazov (Russia) \\
\hline Eva Ciruelos (Spain) & Daniel F. Hayes (USA) & Zhiming Shao (PR China) \\
\hline Marco Colleoni (Italy) & Chiun-Sheng Huang (Taiwan) & Ian Smith (UK) \\
\hline Giuseppe Curigliano (Italy) & Jens Huober (Germany) & Petra Tesarova (Czech Republic) \\
\hline Suzette Delaloge (France) & Zefei Jiang (PR China) & Beat Thürlimann (Switzerland) \\
\hline Carsten Denkert (Germany) & Per Karlsson (Sweden) & Masakazu Toi (Japan) \\
\hline Angelo Di Leo (Italy) & Eun Sook Lee (Korea) & Andrew Tutt (UK) \\
\hline Peter Dubsky (Austria/Switzerland) & Sibylle Loibl (Germany) & Giuseppe Viale (Italy) \\
\hline Bent Ejlertsen (Denmark) & Monica Morrow (USA) & Toru Watanabe (Japan) \\
\hline Florian Fitzal (Austria) & Kent C. Osborne (USA) & Eric P. Winer (USA) \\
\hline \multirow[t]{3}{*}{ Prudence Francis (Australia) } & Olivia Pagani (Switzerland) & Timothy Whelan (Canada) \\
\hline & Ann H. Patridge (USA) & Binghe Xu (PR China) \\
\hline & Martine Piccart-Gebhart (Belgium) & \\
\hline
\end{tabular}

cording to which any of the 4 available multigene signatures - Oncotype DX ${ }^{\circledR}$ Recurrence Score (RS), MammaPrint $70^{\circledR}$ (MP), Prosigna ${ }^{\circledR}$ PAM 50 Risk of Recurrence Score, and EndoPredict ${ }^{\circledR}$ - can be used in Germany (Fig. 1) [1].

\section{Endocrine Adjuvant Therapy}

Endocrine adjuvant treatment is the treatment of choice for patients with ER+ (hormone sensitive) breast cancer [1]. The ideal cutoff value for the use of endocrine therapy is ER $>10 \%$ according to a simple majority vote (38.8\%) of the panelists. Just under $25 \%$ of the panelists indicated that the cutoff is not clearly defined, and 30.1\% saw the cutoff as ER $>1 \%$.

The vote illustrates that there is no ideal cutoff value. From the German point of view, an ER value from $\geq 1 \%$ formally constitutes an indication for endocrine therapy. That is why this situation should be discussed in the tumor board and with the patient, taking into account other factors such as the patient's age and additional prognostic factors.

\section{Premenopause: When Is There an Indication of \\ Ovarian Function Suppression?}

Ovarian function suppression (OFS; GnRH analogues or by bilateral ovariectomy) is an additional op-

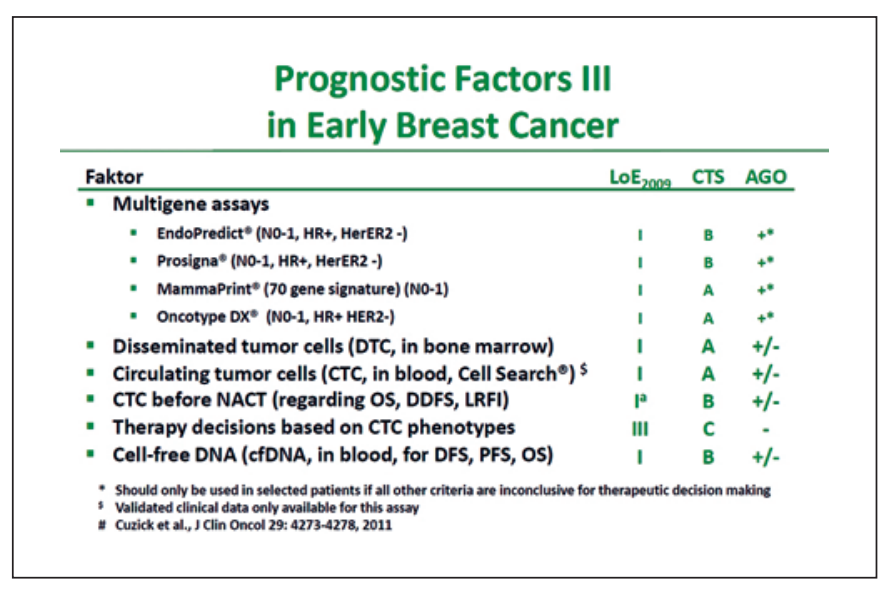

Fig. 1. AGO recommendation 2019 on the use of multigene signatures [1]. With kind permission from AGO Mamma.

tion for endocrine therapy with tamoxifen for premenopausal patients with early ER+ breast cancer. The St. Gallen panelists (68.1\%) and the German expert group consider OFS to be indicated with a high risk of relapse, which also justifies a chemotherapy indication. They also agree that neither a moderate risk without chemotherapy indication nor a positive HER2 status ("by itself") justify OFS.

The German expert group does not agree with the following panelist recommendations: according to the pan- 
elists' majority vote, there is an indication for OFS "by itself" in patients aged $\leq 35$ years $(84.8 \%)$ with a divergent result of multigene expression analysis (59.6\%) and with 1 previously affected lymph node (37.8\%). The German expert group does not agree with these votes due to the following rationale:

- Young age by itself is not usually a mandatory indication for OFS.

- There are currently no data on the reliability of multigene expression analyses in terms of escalating endocrine therapy.

- The crucial requirement for an OFS indication is "persistent ovarian function" following (neo)adjuvant chemotherapy as well as an increased risk of relapse. This is the case if there are 4 positive lymph nodes; with 1-3 positive lymph nodes, it is dependent on further risk factors.

The St. Gallen panelists (55.1\%) and the German expert group agree on the duration of therapy: If indicated, the OFS should ideally be administered for 5 years [7]. If premenopausal patients receive an aromatase inhibitor (AI), additional OFS is mandatory. Under tamoxifen, on the other hand, OFS may be terminated early, if it is necessary due to side effects, for example.

\section{Endocrine Treatment Options under Discussion for \\ Postmenopausal Patients}

The German expert group agrees with the panelists' majority vote $(95.7 \%)$ that postmenopausal patients with $\mathrm{ER}+$ early breast cancer should receive an $\mathrm{AI}$ at some time during their course of therapy. Indications for AI are G3 cancer, a high Ki-67 value, or a positive HER2 status. The German expert group added that the listed factors are not predictive of AI administration but implicate an increased risk of relapse. There is no consensus that an AI should necessarily be given from the beginning ("upfront"). However, upfront administration of an AI is recommended with an increased risk due to advanced tumor stage.

\section{Premenopausal Patients: Therapy after 5 Years of \\ Tamoxifen}

In stage I breast cancer patients who are still premenopausal after 5 years of tamoxifen therapy, endocrine therapy should be discontinued according to a simple majority vote of the panelists (54.3\%). The German expert group agrees but points out that case-by-case decisions may be possible, amongst other things, depending on the patient's safety needs.

There is a consensus that premenopausal patients with stage II breast cancer and lymph node involvement at first diagnosis are at increased risk. Therefore, they should receive tamoxifen for another 5 years (79.6\%). Treatment should not be stopped.

\section{Postmenopausal Situation: Indication of Extended}

\section{Endocrine Therapy}

Endocrine therapy beyond 5 years requires that treatment has been well tolerated in the first 5 years. With this premise, extended endocrine therapy may be an option for patients with stage II ER+ breast cancer.

The majority of panelists agree that the following situations constitute an indication for extended endocrine therapy:

- Stage II patients treated with tamoxifen for the first 5 years regardless of lymph node involvement at first diagnosis (N0: 68.1\%, N+: 97.9\%).

- Stage II patients with lymph node involvement at first diagnosis treated with an AI for 5 years (81.2\%).

The majority of panelists agree that there is no indication for extended endocrine therapy for these patients:

- Stage I patients, regardless of which prior endocrine therapy (tamoxifen or AI) has been given.

- Stage II patients, if there was no lymph node involvement at first diagnosis and if patients had an AI for 5 years.

The German expert group agrees with the voting results. However, they point out that extended endocrine therapy may also be an option in patients at supposedly low risk (stage I or stage II [N0, AI upfront therapy for 5 years]). The treatment decision depends on the risk-benefit ratio and on patient preference after the patient has been thoroughly informed.

Opinions differ about the duration of the extended endocrine therapy: Just under $60 \%$ of panelists voted in favor of extended endocrine therapy for 5 years, equivalent to a total duration of therapy of 10 years. Just under one-third (31.7\%) voted for a total duration of treatment of 7-8 years. From the German perspective, duration of therapy depends on the endocrine pretreatment and should be differentiated accordingly: A total treatment duration of 10 years is an option for patients with 5 -year tamoxifen pretreatment. If patients already had an AI in the first 5 years, extended endocrine treatment is preferably continued for 2-3 years, equivalent to an overall duration of therapy of 7-8 years. If there is a particularly high risk of relapse (e.g., $\geq 10$ positive lymph nodes, G3, etc.), extended endocrine therapy may even be possible for more than 10 years.

\section{Chemotherapy Indication in Patients with Low-Risk Cancer}

According to the majority of St. Gallen panelists (64.6\%), there is an indication for adjuvant chemotherapy in patients with 4-9 positive lymph nodes at first diagnosis. This also applies to luminal A breast cancer (ER+/HER2, G1 with/without low risk of relapse according to multigene expression analysis) and to the "classic" lobular low-risk breast cancer (according to 
multigene expression analysis). The German expert group agrees.

\section{Multigene Signatures in Patients with No Lymph Node Involvement}

This year, the votes on multigene signatures mainly related to prospective studies, such as the TAILORx [8] or MINDACT studies [9]. According to the TAILORx study [8] patients without lymph node involvement and an RS $\leq 25$ do not need chemotherapy. In premenopausal patients ( $<50$ years) with an RS of $21-25$, a small majority (52.1\%) of panelists would consider adjuvant chemotherapy in addition to endocrine therapy with/without OFS. $10.4 \%$ of panelists voted in favor of adjuvant OFS. About a quarter of the panelists voted in favor of adjuvant OFS but saw no indication for chemotherapy.

The German expert group confirms that chemotherapy is indicated in these patients. Patients who continue to be premenopausal after chemotherapy should receive OFS in addition to endocrine therapy. If the patient is clearly postmenopausal, then endocrine therapy without OFS should be given following chemotherapy.

$57.1 \%$ of panelists consider that chemotherapy in postmenopausal patients without lymph node involvement and an $\mathrm{RS} \geq 26$ depends on the other histopathologic criteria, as well as on the patient's preference. The German expert group agrees with this majority vote.

\section{Multigene Expression Analyses in Patients with Lymph \\ Node Involvement}

The Plan B [10] and MINDACT studies [9] validated RS and MP also in patients with lymph node involvement. Taking the results of the Plan B study into account, a majority $(78.7 \%)$ of the panelists consider that adjuvant chemotherapy is not indicated in patients with an RS $<11$ nor in patients aged $>50$ years and with 1-2 positive lymph nodes. From a German perspective, age-related classification is difficult because pre- and postmenopausal patients were randomized in the Plan B study. German experts fail to agree on whether adjuvant chemotherapy is indicated in younger patients ( $<50$ years) with 1-2 positive lymph nodes and low RS $(\mathrm{RS}<11)$. One half voted for and the other half against chemotherapy.

According to the MINDACT study [9], there is no indication for adjuvant therapy in patients at low risk (MP score) with 1-2 positive lymph nodes. The panelists and the German expert group agree that this applies regardless of age, both for patients aged $<50$ years (majority vote: $78.7 \%$ ) and for those $>50$ years (majority vote: $80.9 \%$ ).

\section{Indication for Neoadjuvant Endocrine Therapy}

Postmenopausal patients with a luminal A-like subtype that is confirmed by immunohistochemistry or multigene expression analysis are, in general, candidates for endo-

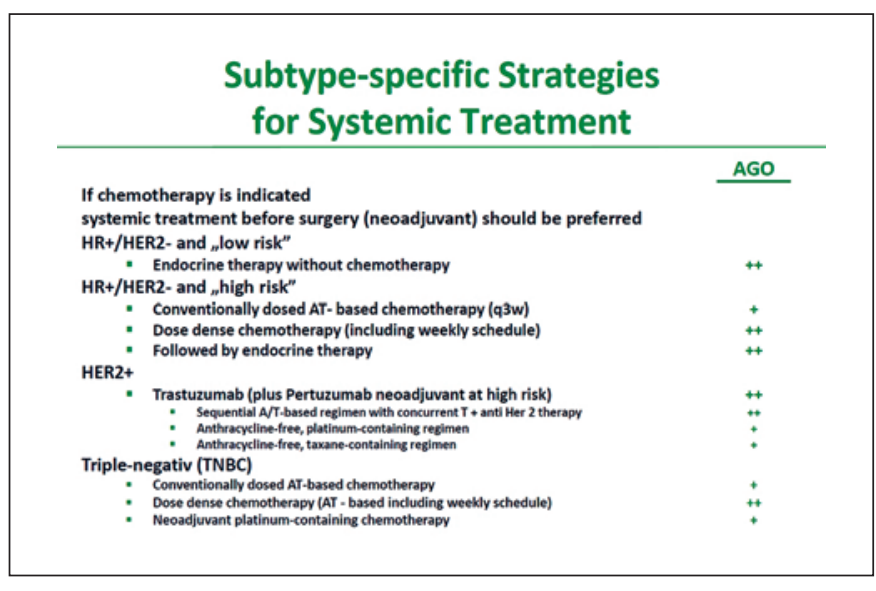

Fig. 2. AGO recommendation 2019 for subgroup-specific systemic therapy in early breast cancer [1]. With kind permission from AGO Mamma.

crine therapy. In the context of neoadjuvant treatment, neoadjuvant endocrine therapy should, therefore, be preferred over neoadjuvant chemotherapy. The German expert group agrees with this majority vote of the panelists (81.2\%). Neoadjuvant therapy should continue until optimal reduction of the tumor burden is achieved. This usually means a treatment duration of more than 6 months.

\section{Adjuvant and Neoadjuvant Chemotherapy}

\section{Chemotherapy in ER+ NO Patients}

Patients with an ER+ breast cancer and no lymph node involvement (N0) should preferably receive chemotherapy containing a taxane plus an alkylating agent, if there is a chemotherapy indication. This majority vote (54.2\%) of the panelists is not consistent with the AGO recommendation [1]. In Germany, anthracycline/taxane-based regimens plus an alkylating agent are preferred. In individual cases - for example, to avoid side effects of anthracyclines - an anthracycline-free regimen, such as the combination of docetaxel/cyclophosphamide, may be given [11].

\section{Chemotherapy in TNBC}

In TNBC, the majority of the St. Gallen panelists preferred an anthracycline/taxane-based regimen plus an alkylating agent already starting from stage I (stage I: 77.6\%; stage II or III: 93.3\%). This is consistent with the AGO recommendation [1]. However, with pTla/b TNBC, the majority of panelists (52.2\%) would not use anthracyclines. According to the AGO recommendations, an alkylating agent/taxane-based regimen with/without anthracycline is an evidence-based option [1]. The German experts add that the neoadjuvant use of chemotherapy is preferable for patients with TNBC due to the verification of response that is possible and important for prognosis. The panelists (61.0\%) 
and the German experts agree that in the (neo)adjuvant setting, anthracycline/taxane-based regimens should preferably be used in a dose-dense manner (Fig. 2) [1].

\section{Neoadjuvant Use of Platinum}

The neoadjuvant use of platinum is a subject of debate in patients with TNBC. A higher rate of pathologic complete response ( $\mathrm{pCR}$ ) is achieved with platinum-based neoadjuvant chemotherapy [12-15]. In the GeparSixto study, the higher $\mathrm{pCR}$ rate for the entire cohort of TNBC patients - irrespective of BRCA $1 / 2$ status - translated into a longer disease-free survival (DFS; $p<0.001)$ [14]. The AGO Mamma recommends the neoadjuvant use of platinum for high-risk patients (high tumor burden, poor response) (Fig. 2). The German expert group, therefore, does not agree with the majority vote of the St. Gallen panelists (56.5\%), who see no neoadjuvant indication for platinum in addition to an anthracycline/alkylating agent/taxane-based regimen.

Moreover, the German expert group does not agree with the majority vote of the panelists (67.3\%) to limit the neoadjuvant use of platinum to patients with BRCA mutation. The BRCA mutation itself is not a predictor of the use of platinum. It increases tumor chemotherapy sensitivity but not specifically the sensitivity to platinum [14].

\section{Chemotherapy for Patients with Small TNBC \\ (pT1apNO)}

Even in a patient with a small, unifocal TNBC without lymph node involvement ( $<6 \mathrm{~mm}, \mathrm{~N} 0)$, chemotherapy may be indicated according to the majority $(65.3 \%)$ of the panelists. From the German point of view, this is a caseby-case decision. German experts see a chemotherapy indication in this situation primarily for young patients with highly proliferative tumors or in patients with a high desire for safety (patient preference).

\section{Votes on HER2-Positive Breast Cancer}

There is a high level of agreement between the votes of the panelists and of the German expert group concerning anti-HER2-directed treatment for HER2-positive (HER2+) early breast cancer patients:

- Patients with HER2+ breast cancer receive anti-HER2 therapy in addition to chemotherapy. This applies from stage I [1] but is not the standard for T1a breast cancers with no lymph node involvement (N0) (majority vote: $55.3 \%$ ).

- For patients with stage I disease, the adjuvant use of trastuzumab with chemotherapy is the standard of care regardless of ER status (majority vote: 61.7\%). The preferred adjuvant regimen is the combination of taxane/trastuzumab in this situation (majority vote: $73.5 \%)$.
- If there is a neoadjuvant indication for trastuzumab in patients with HER2+/ER+ stage I breast cancer, this does not imply that an adjuvant application of pertuzumab is the standard of care (majority vote: $52.1 \%$; $14.6 \%$ abstained).

- Pertuzumab is approved at stage I in patients with lymph node involvement $(\mathrm{N}+)$ and/or negative ER status and is also recommended as such by the AGO Mamma [1]. In contrast, the St. Gallen panelists (majority vote: $48.9 \%$ ) do not consider dual antibody blockade with pertuzumab to be indicated in stage I HER2+ breast cancer. This is not in line with the AGO recommendations [1].

- Patients at stage II with lymph node involvement $(\mathrm{N}+)$ or those at stage III should preferably receive (neo) adjuvant combination therapy with doxorubicin/cyclophosphamide (AC) or epirubicin/cyclophosphamide followed by a taxane plus dual antibody blockade (trastuzumab/pertuzumab) (majority vote: 75.5\%).

- Dual antibody blockade with trastuzumab/pertuzu$\mathrm{mab}$ is the preferred treatment option for all patients at stage II $(\mathrm{N}+$ ) or stage III (majority vote: $76.6 \%$ ).

- If trastuzumab is indicated, duration of therapy is 12 months (majority vote: $89.8 \%$ ).

\section{Indication for Neratinib}

In HER 2+ early breast cancer patients, the adjuvant application of neratinib is an option following (neo)adjuvant trastuzumab. However, in the registration study ExteNET [16], a very selective group of patients benefited from neratinib - those with HER2+/ER+ breast cancer and at least 4 positive lymph nodes. Due to significant toxicity, the adjuvant use of neratinib is seen with caution. Only $21 \%$ of the St. Gallen panelists voted in favor of neratinib for all patients with HR+/HER2+ breast cancer and lymph node involvement, $27 \%$ voted in favor of neratinib in patients with at least 4 positive lymph nodes, and $29.2 \%$ abstained.

The German expert group also refers to the substantial toxicity (diarrhea). Based on the ExteNET study, the AGO Mamma recommends neratinib as a plus/minus (+/-) decision [16], which translates to a case-by-case option [1]. There is no indication for adjuvant neratinib use after dual antibody blockade, as data are lacking.

\section{Tumor Residue after Neoadjuvant Systemic Therapy}

The St. Gallen panelists and the German expert group agree that neoadjuvant systemic therapy is the preferred treatment concept for patients with stage II/III TNBC or HER2 + breast cancer. This applies regardless of whether breast-conserving surgery is feasible or not (majority vote: $98.0 \%)$. The key benefit of the neoadjuvant concept is the verification of response [1]. 
If a residual tumor remains in TNBC after neoadjuvant sequential therapy with $\mathrm{AC}$-taxane, these patients should receive further adjuvant capecitabine $[17,18]$. This recommendation applies regardless of the size of the residual tumor $(\geq /<1 \mathrm{~cm})$ and lymph node involvement $(\mathrm{N}+/ \mathrm{N} 0)$. The German experts agree with the majority vote of the St. Gallen panelists.

Post-neoadjuvant T-DM1 is a new option for patients with HER2 + breast cancer and residual tumor in the breast and/or axilla after neoadjuvant systemic therapy [19]. More than $90 \%$ of St. Gallen panelists as well as the German expert group agree. Neither the size of the residual tumor nor the tumor burden in the axilla is of any importance for the indication of T-DM1. It is also negligible whether patients had received trastuzumab or dual antibody blockade with trastuzumab/pertuzumab in addition to neoadjuvant chemotherapy.

\section{pCR after Neoadjuvant Systemic Therapy}

A point of debate is the adjuvant treatment of patients with HER2+ breast cancer and pCR after neoadjuvant therapy. When patients with lymph node involvement at first diagnosis achieve pCR under neoadjuvant polychemotherapy and dual antibody blockade (trastuzumab/ pertuzumab), a simple majority (47.7\%) of the St. Gallen panelists prefers to continue adjuvant treatment with both antibodies; $38.6 \%$ would only use adjuvant trastuzumab. The German expert group agrees with the majority vote, although this is not yet evidence based. As a rationale, the German experts refer to the tumor biology, which would not be changed after pCR. That is why the increased risk still exists and justifies dual antibody blockade.

From a German perspective, this is also true for patients without lymph node involvement (cN0) at first diagnosis, when both antibodies were used in addition to neoadjuvant polychemotherapy (according to approval in HER2+/ER). In this case, the majority of St. Gallen panelists $(52.2 \%)$ voted in favor of the adjuvant treatment with trastuzumab alone. From a German perspective, de-escalation is not indicated if there is an increased baseline risk (e.g., ER-).

\section{Adjuvant Bone-Modifying Therapy}

Adjuvant use of a bisphosphonate (zoledronic acid every 6 months or daily oral clodronate) in addition to adjuvant endocrine therapy can prolong DFS in postmenopausal patients and improve overall survival. This applies regardless of the bone density [20]. The Austrian ABCSG-12 study [21] shows that the improvement of outcome due to adjuvant bisphosphonates can also be seen in premenopausal patients if they receive concomitant ovar-
Ovarian Protection and Fertility Preservation in Premenopausal Patients Receiving (Neo)-Adjuvant Chemotherapy (CT)

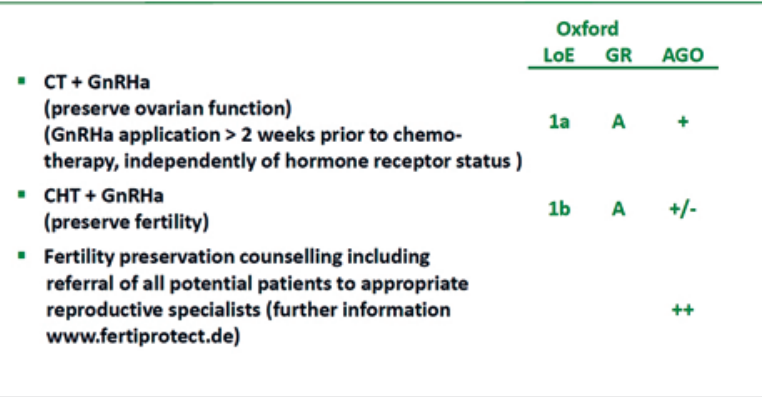

Fig. 3. AGO recommendation 2019 on fertility protection under (neo)adjuvant chemotherapy [1]. With kind permission from AGO Mamma.

ian suppression ( $\mathrm{GnRH}$ analogue) as part of an adjuvant endocrine therapy.

In the context of these data, a small majority (53.1\%) of the panelists supported the concomitant administration of a bisphosphonate (zoledronic acid or clodronate) in premenopausal patients who received an adjuvant $\mathrm{GnRH}$ analogue plus tamoxifen or AI. The rationale is to prolong DFS regardless of bone density.

The German expert group does not agree on this question. It was debated that the indication in favor of a bisphosphonate for DFS prolongation in premenopausal patients is based on only 1 study (ABCSG-12) [21]. The AGO Mamma has thus given a plus/minus rating (caseby-case decision) [1]. The German experts do agree that the osteoprotective effect of bisphosphonates is not linked to the adjuvant indication regarding DFS prolongation.

In postmenopausal patients, the German experts agree with the St. Gallen panelists (83.7\%) that bisphosphonates can be used for DFS prolongation. Interestingly, however, only $42.6 \%$ of the panelists confirmed, in an additional vote, that they implement this in their own clinical practice.

With respect to controversial study data, there is no indication for adjuvant denosumab (60 mg, every 6 months) to prolong DFS. Concerning this point, the German experts agree with the majority vote $(75 \%)$ of the St. Gallen panelists. Unlike the ABCSG-18 study [22, 23], the registration study of denosumab (D-CARE) [23] did not confirm an improved chance for cure with denosumab. However, the D-CARE study also saw a positive impact on the fracture rate [24]. 


\section{Fertility Preservation Should be Offered}

Fertility preservation by suppression of ovarian function should be offered to all patients under (neo)adjuvant chemotherapy who want to have children [1], regardless of the tumor's HR status. The German expert group agrees with the majority vote of the panelists both for patients with HR-negative (91.5\%) and HR-positive breast cancer (79.6\%). This should apply to all patients at childbearing age. The German experts refer to the AGO recommendations for more information (Fig. 3) and to the FertiPROTEKT initiative (www.fertiprotekt.de).

\section{Genetic Testing for High-Risk Mutations}

Genetic testing for high-risk mutations (e.g., BRCA1/2 testing) requires appropriate education and advice for the patient. The German expert group agrees with the majority vote $(70.8 \%)$ of the St. Gallen panelists that such testing should not be considered for all patients, regardless of the familial risk. The panelists' vote whether genetic testing should be recommended to women from high-risk families (clearly positive family history) was unanimous (100\%!). The panelists' vote whether genetic testing should be performed in patients under the age of 35 years at the time of first diagnosis was almost unanimous (95.9\%).

There is consensus that a cutoff by age below 50 years is not suitable to recommend genetic testing without a family history. There is, therefore, no general indication for testing patients younger than 50 years. This is different for patients with TNBC. In this case, the St. Gallen panelists (85.4\%) and the German experts recommend genetic testing if the patient is younger than 60 years at initial diagnosis.

\section{Pregnancy after Breast Cancer}

Breast cancer patients who want to have children should be actively counseled. Endocrine treatment may be interrupted to have a child, if there is no high-risk situation. This should be performed earliest after 18 months (78.0\%). The German expert group, therefore, agrees with the majority vote $(79.2 \%)$ of the panelists that therapy should not be discontinued at any time. Since the benefit of adjuvant endocrine therapy correlates with duration of therapy, the German experts favor a therapy duration of at least 24 months before pregnancy occurs.

Almost $60 \%$ of the St. Gallen panelists recommend that the patient should undergo re-staging prior to pregnancy. The German expert group also recommends re-staging. Regardless of this, the desire to have children is an individual patient decision. The treating physician should educate the patient and constructively support her.

\section{Ductal Carcinoma in situ}

The voting questions posed during the St. Gallen Consensus Meeting for treatment of ductal carcinoma in situ (DCIS) do not adequately reflect the complexity of the situation. In this context, the German expert group agrees with the St. Gallen panelists (97.9\%) that patients with a small DCIS have a more favorable prognosis and need less intensive treatment. This applies especially if the DCIS is detected during screening, the patient is $\geq 50$ years of age, and the DCIS has a favorable low-risk biology.

Favorable prognostic factors and a free margin ( $\geq 5$ $\mathrm{mm}$ ) may support the omission of adjuvant radiotherapy of the DCIS according to the panelists' vote (84.4\%). The question whether to omit adjuvant endocrine treatment was supported by a small majority (55.6\%) of panelists for all DCIS patients and by $42.2 \%$ for DCIS patients with favorable prognostic factors. In patients with favorable prognostic factors, $66.7 \%$ of panelists would omit both adjuvant radiotherapy and adjuvant endocrine therapy.

The German expert group points to the fact that the use of tamoxifen is primarily a preventive intervention to prevent ipsilateral or contralateral secondary breast cancer. The decision to administer adjuvant treatment to a patient with DCIS requires an individual risk-benefit assessment which is guided by various factors, such as tumor size or patient age. No survival benefit has been reported to date for DCIS patients, neither for endocrine therapy nor radiation therapy. The safety margin of $\geq 5$ $\mathrm{mm}$ in the voting questions is arbitrary in the view of the German experts, because no definitive margin is currently defined for DCIS.

\section{Magnitude of Absolute Benefit}

More than $90 \%$ of the panelists voted that breast cancer patients should also be informed of the possibilities and limitations of a therapeutic intervention. Treatment options with only a very limited chance to be successful should be communicated honestly; the option to avoid this treatment should also be discussed with the patient. The German expert group agrees without reservations.

\section{Acknowledgement}

The post-St. Gallen Meeting was organized and implemented by Aurikamed Institute $\mathrm{GmbH}$ and made possible by a grant unconstrained by content from the company Celgene $\mathrm{GmbH}$. The sole responsibility for compiling the manuscript was with the authors of the Writing Committee. The authors would like to thank Birgit-Kristin Pohlmann, Nordkirchen, for editing the manuscript. 


\section{Statement of Ethics}

The authors have to ethical statements to disclose.

\section{Disclosure Statement}

Prof. Diana Lüftner received honoraria from AstraZeneca, Celgene, Pfizer, Novartis, Amgen, Roche, Loreal, Teva, Tesaro, and Eli Lilly. Dr. med. Ingo Bauerfeind has no conflict of interest. Prof. Michael Braun received honoraria from AstraZeneca, Celgene, Daiichi Sankyo, Genomic Health, GSK, Eisai, Genomic Health, GlaxoSmithKline, Medac, Novartis, Pfizer, Puma Biotechnology, Roche, and Teva. Prof. Sara Y. Brucker received honoraria from Novartis, Pfizer, AstraZeneca, and Roche and research funding from Genomic Health. Prof. Peter A. Fasching received funding from Novartis, Cepheid, and BioNTech and honoraria from Novartis, Roche, Pfizer, Celgene, Daiichi-Sankyo, Teva, AstraZeneca, Merck Sharp \& Dohme, Myelo Therapeutics, MacroGenics, Esai, and Puma. Prof. Ricardo Felberbaum, Dr. med. Friederike Hagemann, and Renate Haidinger have no conflict of interest. Prof. Nadia Harbeck received honoraria from Agendia, Amgen, Celgene, Genomic Health, Lilly, MSD, Nanostring, Novartis, Pfizer, Roche, and Sandoz. Prof. Arnd Hönig received honoraria from Amgen, Astra Zeneca, Celgene, MSD, Pfizer, Roche, and Novartis, research funding from Roche and Genentech, and travel grants from Roche, AstraZeneca, Daiichi Sankyo, and Pfizer. Prof. Jens Huober received honoraria from Celgene, Roche, Novartis, Hexal, Pfizer, AstraZeneca, Lilly, Amgen, Eisai, and MSD, research funding from Celgene and Novartis, and travel grants from Roche, Novartis, Celgene Pfizer, und Daiichi-Sankyo. Prof. Christian Jackisch received honoraria from Celgene, Novartis, Pfizer, AstraZeneca, and Roche and research funding from Genomic Health. Prof. Thorsten Kühn has no conflict of interest. PD Dr. Hans-Christian Kolberg received honoraria from Pfizer, Novartis, Roche, Genomic Health, Amgen, AstraZeneca, Riemser, Carl Zeiss Meditec, Teva, Theraclion, Janssen-Cilag, GSK, and LIV Pharma and honoraria for consultancy activity from Pfizer, Novartis, SurgVision, Carl Zeiss Meditec, Amgen, and Onkowissen and holds shares in Theraclion SA and Phaon
Scientific GmbH; he received travel grants from Carl Zeiss Meditec, LIV Pharma, Novartis, Amgen, Pfizer, and Daiichi Sankyo. Prof. Cornelia Kolberg-Liedtke received honoraria from Roche, AstraZeneca, Celgene, Novartis, Pfizer, Lilly, Hexal, Amgen, Eisai, and SonoScape, honoraria for consultancy from Phaon Scientific, Novartis, Pfizer, and Celgene, research funding from Roche, Novartis, and Pfizer, and travel grants from Novartis and Roche. Prof. Nicolai Maass received honoraria from Amgen, AstraZeneca, Celgene, Lilly, MSD, Pfizer, Roche, Novartis, and Daiichi Sankyo. Prof. Toralf Reimer received honoraria from Novartis, Pfizer, Lilly, Celgene, and AstraZeneca. Prof. Andreas Schneeweiss received honoraria from Celgene, Roche, Pfizer, AstraZeneca, Novartis, MSD, Tesaro, and Lilly, research support from Celgene, Roche, AbbVie, and Molecular Partners, and travel grants from Celgene, Roche, and Pfizer. Eva Schumacher-Wulf has no conflict of interest. Prof. Florian Schütz received honoraria for lectures and consultations from Amgen, AstraZeneca, ClinSol, MSD, Novartis, Pfizer, Riemser, and Roche and travel grants from AstraZeneca, Pfizer, and Roche. PD Dr. Marc Thill received honoraria from Amgen, AstraZeneca, Aurikamed, Biom'Up, Celgene, Daiichi Sankyo, Eisai, Genomic Health, Hexal, Lilly, MCI, Medtronic, MSD, Myriad, Neodynamics, Norgine, Novartis, OnkoLive, OmniaMed, pfm Medical, Pfizer, Roche, Tesaro, Teva, RTI Surgical, Onkowissen, Sysmex, and Clovis and research funding from Genomic Health. Prof. Michael Untch received honoraria from the employer (for Ad Board participation, lectures) and travel grants from Amgen, Astra Zeneca, BMS, Celgene, Daiichi Sankyo, Eisai, Janssen Cilag, Johnsen and Johnsen, Lilly Deutschland, Lilly International, MSD Merck, Mundipharma, Myriad Genetics, Odonate, Pfizer, PUMA Biotechnology, Riemser, Roche, Sanofi Aventis, Sividon Diagnostics, and Teva Pharmaceutical Industries Ltd. Dr. med. Rachel Wuerstlein received honoraria from Agendia, Amgen, AstraZeneca, Boehringer Ingelheim, Carl Zeiss, Celgene, Daiichi-Sankyo, Esai, Genomic Health, GSK, Lilly, MSD, Mundipharma, Nanostring, Novartis, Odonate, Paxman, Palleos, Pfizer, Pierre Fabre, Puma Biotechnology, Riemser, Roche, Sandoz/Hexal, Seattle Genetics, Tesaro Bio, and Teva. Prof. Christoph Thomssen received honoraria from AstraZeneca, Celgene, Genomic Health, Novartis, NanoString, Pfizer, and Roche and research funding from NanoString.

\section{References}

1 Ditsch N, Untch M, Thill M, Müller V, Janni $\mathrm{W}$, Albert US, et al. AGO recommendations for the diagnosis and treatment of patients with early breast cancer: Update 2019. Breast Care (Basel). 2019;14:224-45.

2 German Guideline program in Oncology (German Cancer Society, AWMF): Interdisciplinary S3 guideline for early detection, diagnostic, therapy and follow-up of breast cancer, long version 4.0, December 2017, AWMF Registry number: 032-045OL [cited 2019 March 27]. Available from: http://www.leitlinienprogramm-onkologie.de/guidingprinciples/mammakarzinom/

3 Untch M, Thomssen C, Bauerfeind I, Braun M, Brucker SY, Felberbaum R, et al. Primary therapy of early breast cancer: Evidence, controversies, consensus: Spectrum of opinion of German specialists on the 16th St. Gallen International Breast Cancer Conference (Vienna 2019). Geburtshilfe Frauenheilkd. 2019 Jun;79(6):591-604.
4 Balic M, Thomssen C, Würstlein R, Gnant M, Harbeck N. St. Gallen/Vienna 2019: A Brief Summary of the Consensus Discussion on the Optimal Primary Breast Cancer Treatment. Breast Care (Basel). 2019;14:103-10.

5 Schmid P, Adams S, Rugo HS, Schneeweiss A, Barrios $\mathrm{CH}$, Iwata $\mathrm{H}$, et al. Atezolizumab and Nab-Paclitaxel in Advanced Triple-Negative Breast Cancer. N Engl J Med. 2018 Nov;379: 2108-2121.

6 Emens LA, Loi S, Rugo HS, Schneeweiss A, Diéras V, Iwata H, et al. IMpassion130: Efficacy in immune biomarker subgroups from the global, randomized, double-blind, placebo-controlled, phase III study of atezolizumab + nab-paclitaxel in patients with treatment-naïve, locally advanced or metastatic triple-negative breast cancer. In: Proceedings of the 2018 San Antonio Breast Cancer Symposium; 2018 Dec 4-8; San Antonio, TX. Cancer Res. 2019 Feb;79(4 suppl):Abstract nr GS1-04.
7 Pagani O, Regan MM, Francis PA; TEXT and SOFT Investigators; International Breast Cancer Study Group. Exemestane with ovarian suppression in premenopausal breast cancer. N Engl J Med. 2014 Oct;371(14):1358-9.

8 Sparano JA, Gray RJ, Makower DF, Pritchard KI, Albain KS, Hayes DF, et al. Prospective Validation of a 21-Gene Expression Assay in Breast Cancer. N Engl J Med. 2015 Nov; 373(21):2005-14.

9 Cardoso F, van't Veer LJ, Bogaerts J, Slaets L, Viale G, Delaloge S, et al.; MINDACT Investigators. 70-Gene Signature as an Aid to Treatment Decisions in Early-Stage Breast Cancer. N Engl J Med. 2016 Aug;375(8):717-29.

10 Gluz O, Nitz UA, Christgen M, Kates RE, Shak S, Clemens M, et al. West German Study Group Phase III PlanB Trial: First Prospective Outcome Data for the 21-Gene Recurrence Score Assay and Concordance of Prognostic Markers by Central and Local Pathology Assessment. J Clin Oncol. 2016 Jul;34(20):2341-9. 
11 Nitz U, Gluz O, Clemens M, Malter W, Reimer T, Nuding B, et al.; West German Study Group PlanB Investigators. West German Study PlanB Trial: Adjuvant Four Cycles of Epirubicin and Cyclophosphamide Plus Docetaxel Versus Six Cycles of Docetaxel and Cyclophosphamide in HER2-Negative Early Breast Cancer. J Clin Oncol. 2019 Apr;37(10): 799-808.

12 Loibl S, O’Shaughnessy J, Untch M, Sikov WM, Rugo HS, McKee MD, et al. Addition of the PARP inhibitor veliparib plus carboplatin or carboplatin alone to standard neoadjuvant chemotherapy in triple-negative breast cancer (BrighTNess): a randomised, phase 3 trial. Lancet Oncol. 2018 Apr;19(4):497-509.

13 Sparano JA, Zhao F, Martino S, Ligibel JA Perez EA, Saphner T, et al. Long-Term Follow-Up of the E1199 Phase III Trial Evaluating the Role of Taxane and Schedule in Operable Breast Cancer. J Clin Oncol. 2015 Jul; 33(21):2353-60.

14 Hahnen E, Lederer B, Hauke J, Loibl S, Kröber S, Schneeweiss A, et al. Germline Mutation Status, Pathological Complete Response, and Disease-Free Survival in Triple-Negative Breast Cancer: Secondary Analysis of the GeparSixto Randomized Clinical Trial. JAMA Oncol. 2017 Oct;3(10):1378-85.
15 Petrelli F, Coinu A, Borgonovo K, Cabiddu M, Ghilardi M, Lonati V, et al. The value of platinum agents as neoadjuvant chemotherapy in triple-negative breast cancers: a systematic review and meta-analysis. Breast Cancer Res Treat. 2014 Apr;144(2):223-32.

16 Martin M, Holmes FA, Ejlertsen B, Delaloge $\mathrm{S}$, Moy B, Iwata $\mathrm{H}$, et al.; ExteNET Study Group. Neratinib after trastuzumab-based adjuvant therapy in HER2-positive breast cancer (ExteNET): 5-year analysis of a randomised, double-blind, placebo-controlled, phase 3 trial. Lancet Oncol. 2017 Dec;18(12): 1688-700.

17 Masuda N, Lee SJ, Ohtani S, Im YH, Lee ES, Yokota I, et al. Adjuvant Capecitabine for Breast Cancer after Preoperative Chemotherapy. N Engl J Med. 2017 Jun;376(22):2147-59.

18 Zujewski JA, Rubinstein L. CREATE-X a role for capecitabine in early-stage breast cancer: an analysis of available data. NPJ Breast Cancer. 2017 Jul;3(1):27.

19 von Minckwitz G, Huang CS, Mano MS, Loibl S, Mamounas EP, Untch M, et al.; KATHERINE Investigators. Trastuzumab Emtansine for Residual Invasive HER2-Positive Breast Cancer. N Engl J Med. 2019 Feb; 380(7):617-28.

20 Early Breast Cancer Trialists' Collaborative Group (EBCTCG). Adjuvant bisphosphonate treatment in early breast cancer: meta-analyses of individual patient data from randomised trials. Lancet. 2015 Oct;386(10001):1353-61.
21 Gnant M, Mlineritsch B, Stoeger H, LuschinEbengreuth G, Heck D, Menzel C, et al.; Austrian Breast and Colorectal Cancer Study Group, Vienna, Austria. Adjuvant endocrine therapy plus zoledronic acid in premenopausal women with early-stage breast cancer: 62-month follow-up from the ABCSG-12 randomised trial. Lancet Oncol. 2011 Jul 12(7):631-41

22 Gnant M, Pfeiler G, Steger GG, Egle D, Greil $R$, Fitzal F, et al. Adjuvant denosumab in postmenopausal patients with hormone receptorpositive breast cancer (ABCSG-18): diseasefree survival results from a randomised, double-blind, placebo-controlled, phase 3 trial. Lancet Oncol. 2019 Mar;20(3):339-51.

23 Gnant M, Pfeiler G, Dubsky PC, Hubalek M, Greil R, Jakesz R, et al.; Austrian Breast and Colorectal Cancer Study Group. Adjuvant denosumab in breast cancer (ABCSG-18): a multicentre, randomised, double-blind, placebo-controlled trial. Lancet. 2015 Aug; 386(9992):433-43.

24 Coleman RE, Finkelstein D, Barrios CH, Martin M, Iwata H, Glaspy JA, et al. Adjuvant denosumab in early breast cancer: first results from international multicenter randomized phase III placebo controlled D-CARE study. J Clin Oncol. 2018;36(15 suppl):501-501. 